\title{
Quality Requirements for Multimedia Interactive Informative Systems
}

\author{
Sylviane Levy ${ }^{1}$, Fernando Gamboa ${ }^{2}$ \\ ${ }^{1}$ Dirección General de Cómputo y de Tecnologías de Información y Comunicación, Universidad Nacional Autónoma de México, \\ México D.F., México; ${ }^{2}$ Centro de Ciencias Aplicadas y Desarrollo Tecnológico, Universidad Nacional Autónoma de México, \\ México D.F., México. \\ Email: sylviane@unam.mx
}

Received May $28^{\text {th }}$, 2013; revised June $30^{\text {th }}, 2013$; accepted July $8^{\text {th }}, 2013$

Copyright (c) 2013 Sylviane Levy, Fernando Gamboa. This is an open access article distributed under the Creative Commons Attribution License, which permits unrestricted use, distribution, and reproduction in any medium, provided the original work is properly cited.

\begin{abstract}
Multimedia Interactive Informative Systems (MIIS) are software applications resulting from the convergence of multiples technologies such as audiovisual, computing and communication. They aim to transmit information to a large, diverse and dispersed public. As with other mass media, the fulfillment of MIIS goals depends largely on the quality of communication between the system and end users. Therefore, those systems should be developed in order to ensure that this quality requirement is satisfied. If MIIS are constructed according to usual software engineering practices, the analysis discipline of the development process includes requirements identification and specification; however, these techniques are focused on functional requirements, and they do not give much importance to non-functional requirements. In this paper, we propose a development process based on the production of videogames which has two different phases: preproduction and production. The first phase, corresponding to requirements identification, derives into the concept of system. In order to translate this concept into a specification, we propose the use of new communicational attributes and a MIIS metamodel. The establishment of MIIS non-functional specification is the result of analyzing class diagrams through quality attributes. In order to evaluate if the specifications are responding to communicational attributes, a functional prototype is built and evaluated with end users. The proposed methodology is applied to a real case study.
\end{abstract}

Keywords: Multimedia Interactive Informative Systems; Multimedia; Quality Model in Use; Quality Requirements; Metamodeling

\section{Introduction}

Multimedia Interactive Informative Systems (MIIS) are software systems that result from the convergence of multiple technologies, for example the combination of computational, audiovisual and communication technologies. The purpose of MIIS is to diffuse information to a large, dispersed, diverse, and non-captive public. An example of a MIIS would be a website whose goal is to transmit information, such as an online newspaper or a cultural website. A kiosk in a museum, cultural CDROMS, and many applications for mobile phones and tablets can also be considered as MIIS.

As with other communication media, such as cinema and television, whether or not the MIIS fulfill its goals depends largely on quality characteristics related to the communication between the transmitter and the receiver. In this case, the qualities of communication between the
MIIS and its final users are essential, and the success of the MIIS depends on the user finding these systems attractive, interesting, informative, and credible. However, these quality requirements are not included among the attributes of quality models used for software such as the ISO/IEC norm 25010 [1] and its predecessor ISO/IEC 9126.

These types of non-functional requirements are difficult to translate into specifications. But if they are not satisfied, the user can discard the application with the same freedom that she or he can change the channel of the television or leave a cinema when the film is disappointing, rendering the system essentially useless.

Existing models of quality barely consider these characteristics. Software applications traditionally help captive users to solve concrete problems, and there is no need for retaining their interest. The MIIS's user, on the 
other hand, is generally non-captive since its acquisition of information is a voluntary decision and not bound by the necessity to use the system.

Traditionally, the specifications of a computer system are guided by functional requirements expressed by clients and final users. In the MIIS' case, where the objective is to transmit information, the users cannot easily decide on the content of the application and therefore there is a need of intervention from experts for deciding what information should be contained in the system [2].

The above observations explain why it is necessary to explore new methodologies aiming to establish the specifications of a MIIS, and to define new quality requirements more appropriate to evaluate the quality of the transmission of knowledge to non-captive users.

This study proposes a methodology for MIIS specification based on: 1) A communicational analysis that allows the definition of a creative concept of the system to be built; 2) A metamodel of MIIS to be used for designing the system; 3) New characteristics that enrich the model of quality in use ISO/IEC 25010, in order to analyze MIIS communicational aspects; and 4) A prototyping-centered methodology for designing a MIIS.

This paper is organized as followed: Section 2 summarizes existing methodologies which served as background of our work; Section 3 presents Multimedia Interactive Informative Systems (MIIS) as a metamodel; Section 4 defines the expected qualities for MIIS; the proposed methodology is developed in Section 5 and a case study is detailed in Section 6; Section 7 is the conclusion.

\section{Background}

Studies covering the development of multimedia have split along two general lines:

The first line derives from the field of communication, where the authors concentrate on the creation of a new communication medium. These works are based on a development process strongly influenced by film and video making.

The second line comes from the design and development of conventional software. It focuses on Requirement Engineering and on the resolution of problems linked to the handling of multiple media, but leaves aside the communicational problem.

\subsection{Communication Perspective}

In general, the works coming from the field of communication studies are prepared as scholarly manuals and they base their conceptual and development methodology on other media, such as cinema or television.

One of the most mature of these works is by Friedmann, who proposes a process of analysis and scriptwriting for different means of communication, called visual media, which include traditional media but also websites, educational and training software applications, information kiosks, and videogames [3]. Friedmann establishes an analytical and creative process whose goal is to yield a creative concept of visual media on the basis of which the script is written.

Nonetheless, even in the case of interactive systems, the screenplay is written in a style strongly influenced by the cinematographic industry, given that: "Books, movies, television, theatre-all imply the creation of specific documents that establish formats that the interactive Industry does not have" [3]. In particular, Friedmann does not specify how the script can be converted to the specifications of a system that must be understood, interpreted, and developed by a multidisciplinary team of designers, communication specialists, and programmers, among others.

This study will use Friedmann's framework to analyze the domain of MIIS' problem and to propose a creative concept to be translated into specifications of system.

\subsection{Software Perspective}

In the field of computing, there are few studies which consider the transmission of information as the main purpose of the system. Among the most original studies are those from Bolchini [4], who proposed the term "infosuasive" to define Web applications that are both informative and persuasive, such as those applications that "aim at supporting knowledge needs and have also the (declared or not declared) goal of influencing user's opinions, attitudes and behaviors.” Bolchini based his work on the Objective-Centered Design Cooper's methodology [5], centering his analysis on the need to meet the objectives of different users and introducing communication objectives as elements of analysis for the design of Web applications. One of his contributions was to identify the need to carry out and to document a communication analysis to build website requirements, such as content, layout, architecture, and navigation.

Another work which considers new types of requirements is offered by Davide Callele [6], who studies video games in particular and has introduced the concept of emotional requirements which analyze and document the emotions to be transmitted to the users/players. On the basis of these emotional requirements, he establishes functional and non-functional requirements. The concepts presented in these works will allow us to analyze the requirements of MIIS through qualitative requirements such as attractiveness, interestingness, informativeness and credibility.

There are a variety of techniques to elicit requirements [7], which generally involve the system's end-users and stakeholders. But there are very few studies that consider 
requirements not elicited by users or stakeholders, as needed in the case of MIIS.

Among these studies, a combination of production inspired by movie realization and software engineering is used in video game industry [8]. The development process of a video game is composed by two phases: preproduction and production. The first one results in a Game Design Document (GDD), which is a creative work written by game designers. The second one is a traditional development process. Transition between both phases is particularly difficult since it is necessary to translate the GDD into a specification: "Despite the recognized need, we have discovered no evidence that a process for managing the transition from preproduction to production has been proposed (recognizing that such a process may exist within an organization but remain unreported in the literature) [8]."

In this work, we will follow the video game development process proposed by [8]: the first phase, the concept of MIIS will be created and, in order to move from concept to a traditional software engineering process, we propose the use of a metamodel to represent the system and derive its specifications through the analysis of quality attributes.

\section{Multimedia Interactive Informative Systems (MIIS)}

\subsection{Definition}

In 1999, Burleson [9] defines a "multimedia system to be a computer-based communication system which delivers heterogeneous and compressed/coded/encrypted content (text, audio, video, graphics) from a source or storage device and transfers it over a heterogeneous channel (Internet, wireless network, local area network) to an end-user while maintaining perceptual integrity”. In this definition, Burleson points out the communicational character of multimedia system as well as the result of multiples technologies as computing, communication and audiovisual. Other authors have tried to define those systems. For example in [10], multimedia is defined as "a computer system with the capacity to deliver visual and audio information to a user interactively” and Guéneau describes a digital creative project through three components: visualization, interactivity and content [11].

Based on these definitions, we define a MIIS as an informative communication system, resulting from the convergence of multiples technologies, delivering some content to an end user in a multimedia environment and interactively.

In this definition, we point out the communicational and informative character of MIIS as well as its components.

\subsection{MIIS Metamodel}

Based on this definition, we propose a metamodel to describe a MIIS. The Metamodel is composed by four main metaclasses, WINDOW, CONTENT, SCENE and DATA (Figure 1).

WINDOW metaclass is an abstract class composed by SCENE and CONTENT metaclasses. Both of them are composed by several media, represented by the MEDIA metaclass. This metaclass is associated with INTERACTION saying that media are to be interactive. Finally, the metaclass DATA contains users and system data.

- Window represents what is between the system and users, what users see, hear and interact with. They are linked between them with a *..* multiplicity which indicates that one window is linked with many windows and many windows are linked to 1 , allowing navigation all over the system. It is composed by two metaclasses:

1) Content represents the information to be transmitted as well as the way to be delivered. It is displayed by different media, which could be simply audiovisual or also interactive.

2) Scene represents all the audiovisual elements of the interfaces that have the purpose of involving the user into the application.

- Data contains all information which is not visible by users but necessary for MIIS as system and users databases.

- Media can represent information, contextualize content, have an esthetic, emotional, or functional pur-

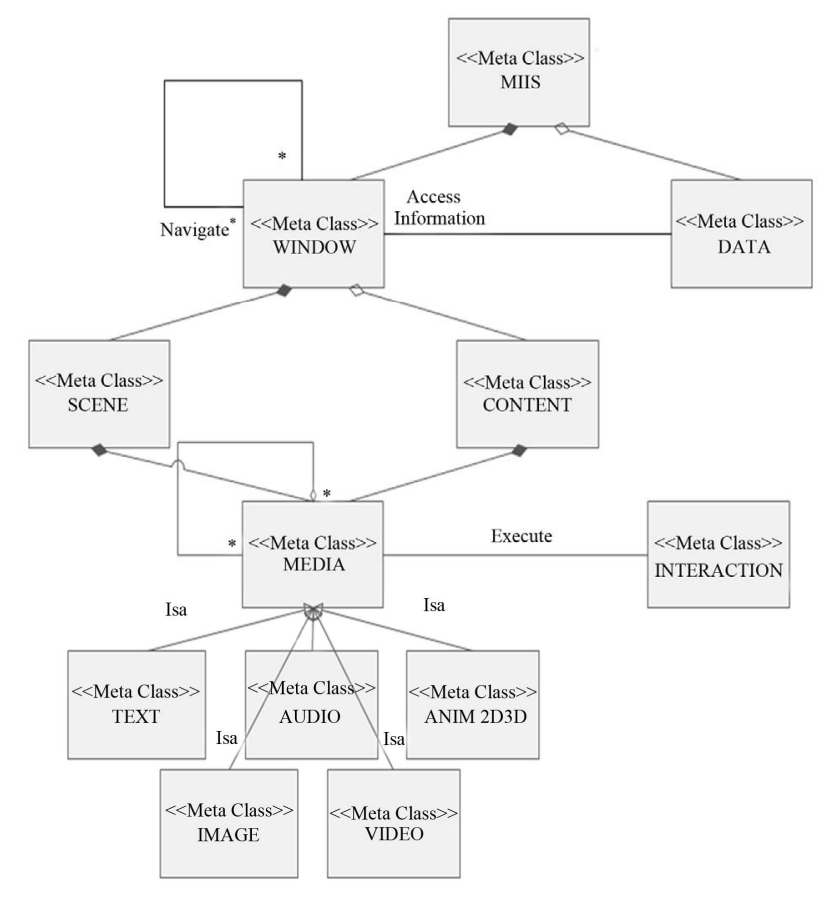

Figure 1. MIIS metamodel. 
pose. They can be interactive or just audiovisual elements. Media are made by classes of text, images, sounds, videos, 2D and 3D animations.

It is important to point out that, on the basis of these concepts, Interactive media are media with a code associated which allows any kind of action, particularly users' interaction with system.

\section{MIIS Quality}

\subsection{Quality of a Software}

Among the models of quality that are most often used to evaluate software is the standard ISO 25010 which substitutes the ISO 9126 standard for the models of quality of software [1] and ISO 25012 [14] for the quality of data.

The ISO/IEC 25010 standard [1] defines Software Product Quality and In Use Quality Models, which have proven to be useful tools to specify Non Functional Requirements (NFR). According to ISO/IEC 25010:

- A product quality model is composed of eight characteristics (which are further subdivided into subcharacteristics) related to static properties of software and dynamic properties of the computer system. The model is applicable to both computer systems and software products.

- A quality in use model is composed of five characteristics (some of which are further subdivided into subcharacteristics) related to the outcome of interaction when a product is used in a particular context of use. This system model is applicable to both computer systems and software products in use.

The characteristics of the product quality are considered during the development of the system (Internal/External quality). Internal quality is specified and measured during the development process and external quality is observed while testing the not yet delivered system. The Internal/External quality is specified and measured by the developers' team; they affect the quality in-use characteristics, which are directly perceived by the end user. It means that during the construction of the system, the Internal/External quality must be considered to build the desired quality into the final system.

The quality of a system is defined as the degree to which the system satisfies the stated and implied needs of its various stakeholders, and thus provides value [1]. Quality models represent these needs; they categorize the product quality into characteristics, which can be further subdivided into sub-characteristics; these can be refined until the quality attributes or measurable elements are obtained. The ISO/IEC 25010 standard offers different quality models for the software product; in this context, we are interested in Quality in Use Model.

Traditionally, requirements of a computer system are established based on an analysis of the needs expressed by clients and/or final users. In the case of MIIS, where the objective is to transmit information, the clients, for example academics, teachers or art institute managers, cannot easily decide on the content of the application and its treatment.

This task therefore requires the participation of experts in communication and information content, since they must decide what information should be contained in the system [2].

In this paper, the modeling of the quality of MIISs is proposed, as a necessary step towards a systematic design of these systems involving the knowledge of the expert on the domain. The main goal of this work is to present the MIIS quality modeling by adapting the ISO/IEC 25010 quality model to specify the communicative quality of MIISs. The results can be used to improve the requirements elicitation discipline in the development process of MIIS.

\subsection{Quality of a MIIS}

As we can observe, among the characteristics of the quality in use model, none of them address the communicational aspects of the software, which express the quality of the communication established with the user. In other informative communication media such as documentary film, many studies have analyzed how to conceive and qualify a good film. In [12], the author describes for example some of the most important characteristics of a documentary. In author's opinion, the first objective of a production is to catch attention and, once audience's attention is caught, to maintain it until the end. To attract this attention, it is necessary to implicate the viewer from a cognitive and also from an emotional point of view: "Implication is possible when audience feels that the experiences that a documentary is transmitting are authentic" [12].

From those ideas, we propose to introduce 4 new subcharacteristics: attractiveness, interestingness, informativeness, and credibility.

\subsubsection{Definition of Communicational Quality Characteristic}

If a system of quality is to be characterized by its level of communication, the model of quality in use described in [1] should be complemented by a new characteristic, whose goal is to qualify the quality of communication between the system and its users. Four sub-characteristics are introduced as follow:

- Attractiveness is the capacity of the system or its components to catch and maintain user's attention for a minimum time in a specific context.

- Interestingness is the capacity of the system or its components to maintain user's attention on informa- 
tion that is being transmitted.

- Informativeness is the capacity of the system or its components to inform the user with relevance, understanding and structured way, in a specific context.

- Credibility is the capacity of the system or its components to allow the user to feel involved in a credible and authentic environment in a specific context. It allows the user to believe in the veracity of the transmitted information.

\subsubsection{Evaluation of Communicational Quality Characteristic}

The evaluation of the characteristic of communication of a MIIS is generally used to assess the impact of its attributes on the user before a complete (and expensive) development of the application. Typically, this evaluation is done through a prototype which includes the most important windows responsible of assuming the communicational attributes.

In [13] the author affirms that "Assessment methods can be divided into two groups: inspection methods and test. Inspection methods are used in order to test compliance of a system with the standards and guidelines for quality assurance”. Among these standards, heuristic evaluation is the most often used by experts to determine if the 10 heuristics proposed by Jakob Nielsen [14] are complied.

In this work, we are interested about the second group, which consists of test methods used with end-users to obtain feedback on whether the system meets the predefined quality attributes or not. The most common form of user testing is the use of checklists or questionnaires.

Checklists can include: the importance of sources, grammar and orthography, correctness, relevance and actuality of information.

Questionnaires allow to measure subjective attributes as "Attractiveness, Interestingness, Informativeness" or Credibility through qualitative criteria. Some quantitative criteria may be defined. They include: time spent by users, number of windows visited and number of media displayed or information retained by users.

In Table 1, some examples of criteria are shown, related with communicability attributes and, as suggested in [15], in order to evaluate the proposed set of attributes, we propose some metrics.

\subsubsection{Communicational Metrics}

Communicational metrics measure the extent to which users feel if the product is attractive, interesting, and credible and transmit information efficiently. Metrics are used to evaluate each attribute. A balanced project should meet the four attributes in the same proportion. In Table 2, we define some metrics that will be used in the case study.
Table 1. Examples of evaluation criteria.

\begin{tabular}{|c|c|c|c|}
\hline $\begin{array}{c}\text { Quality } \\
\text { attributes }\end{array}$ & Checklist & $\begin{array}{l}\text { Quantitative } \\
\text { criteria }\end{array}$ & $\begin{array}{l}\text { Qualitative } \\
\text { criteria }\end{array}$ \\
\hline \multirow[t]{3}{*}{ Attractiveness } & & $\begin{array}{c}\text {-Total time spent } \\
\text { by users }\end{array}$ & $\begin{array}{c}\text {-Curiosity about } \\
\text { system }\end{array}$ \\
\hline & & $\begin{array}{c}\text { •Number of } \\
\text { windows visited }\end{array}$ & -Interest to go on \\
\hline & & $\begin{array}{l}\bullet \text { Number of } \\
\text { media displayed } \\
\text { by users }\end{array}$ & \\
\hline \multirow[t]{2}{*}{ Interestingness } & & $\begin{array}{l}\text { •Time spent in } \\
\text { each Window }\end{array}$ & -Interest about \\
\hline & & $\begin{array}{l}\bullet \text { Information } \\
\text { retained by users }\end{array}$ & content \\
\hline \multirow{2}{*}{ Informativeness } & $\begin{array}{l}\text { •Information } \\
\text { relevance }\end{array}$ & $\begin{array}{l}\text { •Information } \\
\text { understanding }\end{array}$ & \\
\hline & $\begin{array}{c}\text {-Information } \\
\text { actuality }\end{array}$ & $\begin{array}{l}\text { •Key information } \\
\text { retained by users }\end{array}$ & \\
\hline \multirow{4}{*}{ Credibility } & •References & & $\begin{array}{l}\text { •Credibility } \\
\text { about scenes }\end{array}$ \\
\hline & $\begin{array}{l}\text {-Source of } \\
\text { information }\end{array}$ & & $\begin{array}{l}\cdot \text { Credibility } \\
\text { about } \\
\text { information }\end{array}$ \\
\hline & $\begin{array}{l}\text {-Correctness of } \\
\text { information }\end{array}$ & & \\
\hline & $\begin{array}{l}\text { •Grammar and } \\
\text { orthography }\end{array}$ & & \\
\hline
\end{tabular}

To achieve the required quality of communication, the components of the system must be designed with that intention in mind. In other words, they must be the product of a requirements analysis that includes the application of communicational purpose.

\section{MIIS Development Process}

As mentioned before, we will follow the development process used in video game industry, which has two different phases: preproduction and production. The first one delivers MIIS' concept that has to be transformed into specification which therefore allows, a multidisciplinary team to develop it. The second is a traditional software process, generally iterative and incremental, resulting in a MIIS. In this work, we are interested in the transition from preproduction to production.

MIIS' concept is the product of a communicational analysis and a creative process.

\subsection{Communicational Analysis}

Communicational analysis is based upon the different elements, which compose the domain of the problem. Friedmann [3] suggests that, in order to write the scripting of a visual media, it is necessary to define the communication problem, the target audience, the objective and the content. 
Table 2. Some communicational metrics.

\begin{tabular}{|c|c|c|c|c|}
\hline Metric name & Purpose of the metric & Method of application & Measurement & Interpretation \\
\hline $\begin{array}{l}\text { Attribute } \\
\text { questionnaire }\end{array}$ & $\begin{array}{l}\text { How (attractive, interesting, credible) } \\
\text { is a Window for the user? }\end{array}$ & User test & $\begin{array}{c}X=\sum(A i) / n \\
A_{i}=\text { response to a question } \\
n=\text { number of responses }\end{array}$ & The larger the better \\
\hline Window time & $\begin{array}{l}\text { How long does the user spend in each } \\
\text { Window? }\end{array}$ & User test & $\begin{array}{c}X=\sum(T i) / t \cdot n \\
T_{i}=\text { time spent by users in a Window } \\
t=\text { expected time } \\
n=\text { number of users }\end{array}$ & $\begin{array}{l}X>1 \text {, is above expectation } \\
X<1 \text {, is under expectation }\end{array}$ \\
\hline
\end{tabular}

The definition of communication problem tries to focus on what is the need to produce a MIIS: "Our axiom states that every program is a response to a communication problem. If there were no need to show, tell, explain, attract, entertain, seduce, delight, or distract an audience, there would be no reason to make a program and, therefore, no need to write a script" [3].

The definition of target audience tries to sharpen a public, even if it is potentially wide, diverse and dispersed. There is always a target public which allows focusing on a specific age, gender, cultural level, nationality, socioeconomic level, etc.

The objective is closely associated to the communication problem: it states what problem the MIIS will help. Generally, the objective is easy to see. The difficulty is how to reach it.

The content, as part of the communication analysis, should include the main themes to be treated. Their investigation and documentation is part of the following process.

\subsection{Creative Concept}

The analysis of those elements should lead to define a strategy: On Friedmann's opinion [3], “to write a successful script that solve the communication problem, we need to figure out how to achieve the objective, reach the target audience, and suggest the content that leads to effective communication”. This strategy could be one, already used in other successful systems with similar problem domain, or can be an original one.

As a result of choosing a strategy, it is applied to a specific problem domain, and inventing a creative concept.

The purpose of the creative concept is to "solve the communication problem, reach the target audience, achieve the objective, embody the strategy, provide the content of the application, and show how it will work" [3]. It also should respond to the quality characteristics stated in the extended quality model, specifying how the system will be attractive, interesting, informative and credible to end users.
This creative concept should be translated or specified in terms of an instance of the MIIS metamodel.

\subsection{MIIS Model}

Creative concept should be translated in terms of media and interactive media. The role of MIIS metamodel, just as DSL (Domain Specific Language), is to guide this process:

The first step is to define the different windows and navigation map, which shows the way they are linked.

The second step, defines scene and content for each window: scenes represent all the audiovisual components and content contain the information to be transmitted. In order to insure that quality characteristics are considered by the MIIS model, it is necessary to decide which component will take in charge each one of them.

\subsection{MIIS Specification}

According to [16], “Quality is a feature that is 'built' in an information system and not added afterwards.” In this sense, if we accept that a MIIS should be attractive, interesting, informative and credible, in order to be used and appreciated by its end users, the designer must be able to incorporate these goals into the MIIS.

It is then necessary to decide which window will assume one or more attributes and specify how media assume the corresponding attribute, establishing specifications for each of them. In this way, it is possible to trace problems through evaluations during the process of production.

\section{Case Study}

In order to illustrate the above, we will apply the methodology to a case study: "Pierre y la Coatlicue", a learning system for Spanish language and Mexican culture. The first step is then to carry out a communicational analysis, as suggested by Friedmann [3].

\subsection{Communicational Analysis}

In the National University Center for Foreigners in Mex- 
ico, students must follow classes on Spanish and Mexican culture. If the students are captive in the study of the language, they are not necessarily captive in the study of Mexican culture since their priority is generally to learn Spanish and not in learning Mexican culture. Besides, teachers on cultural issues are not always familiar with teaching foreigners and are having problem in communicating with them.

Therefore, the main target is foreign students at National University and their teachers. Since the system will be on line, it can be used by everybody interested in learning Spanish and Mexican culture.

The objective of the system is then how to motivate foreign students to learn and understand Mexican culture while learning Spanish.

Information requirements are provided by professors and are composed by texts with exercises and grammar texts.

\subsection{Creative Concept}

The strategy selected is the use of narrative. Telling a story has the virtue of motivating the user to know what is going to happen next, and therefore maintains his or her interest in the application $[17,18]$.

On the basis of this strategy the following concept was proposed:

Tell a story through several stages in which the main character is a foreigner visiting Mexico City with whom students can identify and feel empathy towards. The inciting element of the story is the search for the Coatlicue, an Aztec monolith, in different locations of Mexico City. The story must include elements of mystery and plots to maintain the interest of the user and incite him/her to pursue. A set of virtual resources, which young people are familiar with, will be offered for transmitting the information, such as a mobile phone to communicate with family and experts, a camera, a GPS to move around the city, a music player, interactive books, and a Facebook link to communicate with other students. The credibility of the information is established by the creation of a realistic context and characters.

This concept specifies the manner in which the system will respond to questions such as: how to attract and maintain interest, and how to inform and involve the user.

\subsection{Pierre y la Coatlicue Model}

In order to model Pierre y la Coatlicue MIIS, the first step consists in selecting the different windows of application: Registration, different locations visited by Pierre, Mobile phone composed by Calls, Camera, GPS and Music Player, Spanish and History Books and a Field Diary.

In the second step, each window is modeled as an in- stantiation of MIIS metamodel, deciding which media compose his scene and content. This model can be represented through a table instead of diagram. In Table 3, First location (Zócalo) is represented as an example. Zócalo location is composed, as all windows, by scene and content, which are composed by different media.

This model allows graphic designer to propose a first sketch (Figure 2). In order to apply a graphical style to the sketch, non functional requirements specifications are needed.

\subsection{Pierre y la Coatlicue Requirements Specification}

In order to traduce the concept into specifications, it is necessary to decide in first place, which Windows would assume communicational attributes. In Figure 3, those attributes are associated to each window.

Table 3. Zócalo model.

\begin{tabular}{|c|c|c|}
\hline Zócalo & Scene & Content \\
\hline Texts & Localization & \\
\hline Images & $\begin{array}{ll}\text { - } & \text { Zócalo } \\
\text { - } & \text { Cars } \\
\text { - } & \text { Walkers } \\
\text { - } & \text { Typical persons }\end{array}$ & \\
\hline Audio & $\begin{array}{ll}\text { - } & \text { Cars } \\
\text { - } & \text { Bus } \\
\text { - } & \text { Zócalo sounds }\end{array}$ & $\begin{array}{l}\text { Dialogue between } \\
\text { Pierre and walker }\end{array}$ \\
\hline Animations & $\begin{array}{ll}\text { - } & \text { Sky } \\
\text { - } & \text { Pierre }\end{array}$ & $\begin{array}{l}\text { Pierre arriving at } \\
\text { Zócalo, discovering } \\
\text { Coatlicue plate and } \\
\text { talking with walker }\end{array}$ \\
\hline Interactive Media & \begin{tabular}{ll} 
& \multicolumn{1}{c}{ Buttons: } \\
- & Display animation \\
- & Ticket \\
- & Menu \\
- & Exit
\end{tabular} & \\
\hline
\end{tabular}

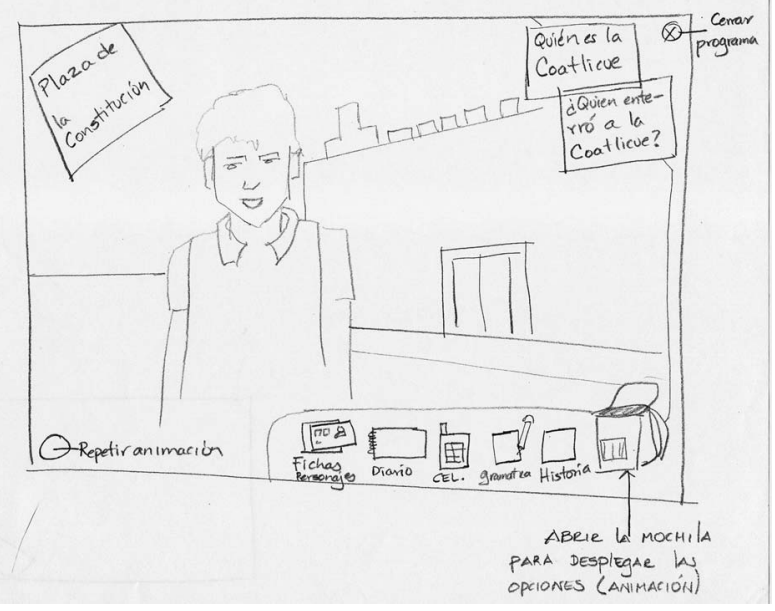

Figure 2. Zocalo sketch. 


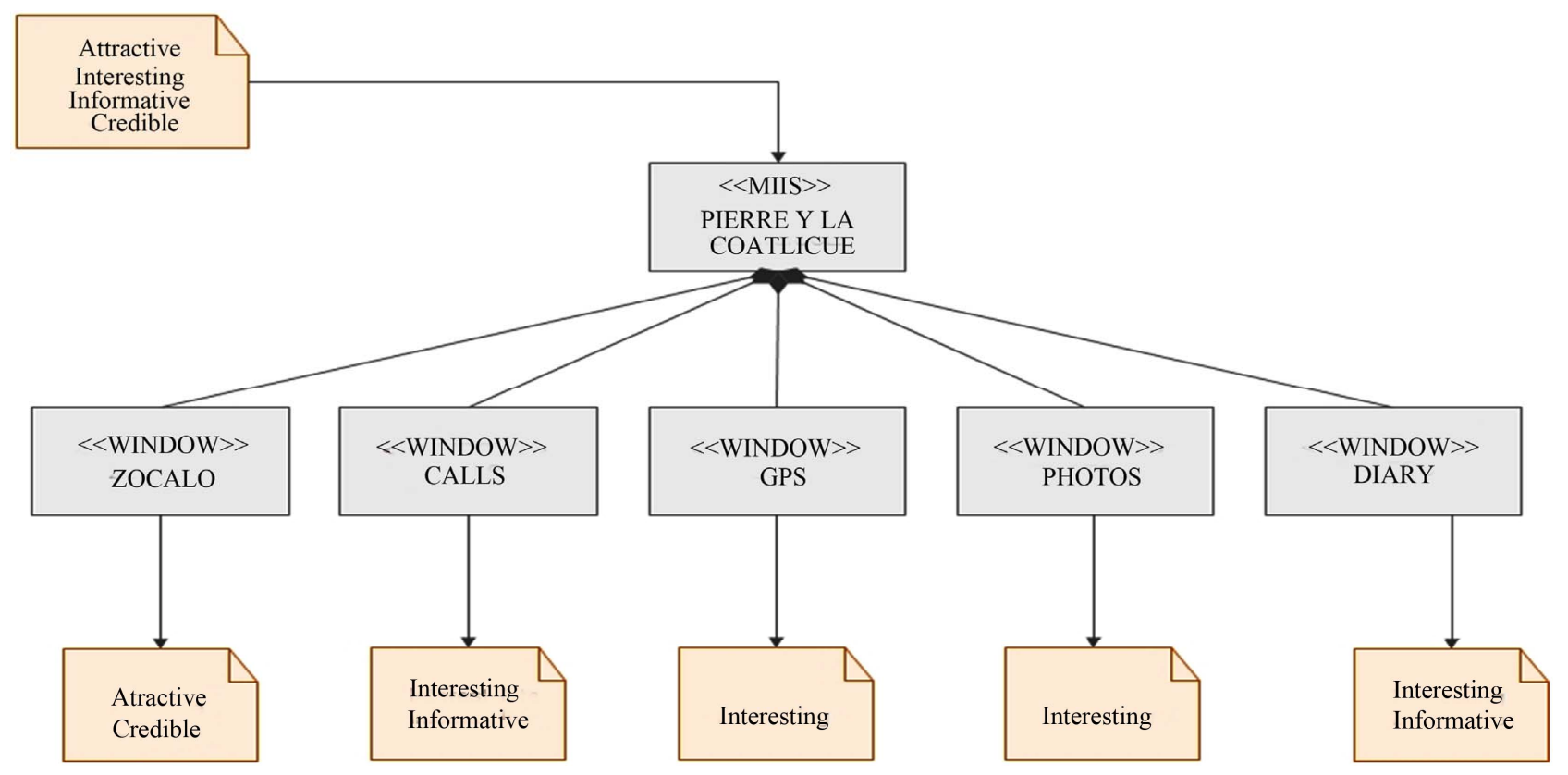

Figure 3. Communicational attributes assumed by each window.

As an example, in Table 4, we established specifications requirements for window Zócalo, the first location Pierre visits.

\subsection{Evaluation of Communicational Attributes}

In order to evaluate if the specifications are responding to communicational attributes, a functional prototype is built, following the process suggested in [19], with the main windows and media that will allow evaluating them. Since it is not possible to evaluate every attribute in a prototype, our main interest is to evaluate student's attractiveness, interest and comprehensiveness of the story. Books and interactive activities were not evaluated as they are part of teacher's requirements, and based on their pedagogical theory and experience.

In Table 4, we synthesized the prototype that we built, composed by windows and media that should allow carrying on the evaluation. Criteria of evaluation proposed in Table 1, are then associated to each Window and applied to questionnaires in order to evaluate communicability attributes (Table 5).

Attractiveness questions are used to assess student's interest to go forward and imagine the rest of the story. If the story is too predictable, there is less interest to go forward.

Interestingness questions are asked in order to assess student's interest about story and information transmitted

Credibility questions are used in order to assess student's credibility of characters, scenes and story situation.

Informativeness questions are used in order to assess student's understanding of information contained in the story.

Time spent by users in each Window, is compared to the minimum time to navigate around, established by us.

In order to carry on the evaluation, teachers used the system in their classroom and after, the students were asked to freely use the system, as they were home. All the session was recorded through "Camtasia" and, then students responded to the questionnaire. They were asked for each window to give a mark between 1 and 5 ( 5 is the highest) and a comment, and the system recorded the time spent on each of them. Table 6 shows the results of these questionnaires.

\subsection{Interpretation and Proposals}

Zócalo window is composed by the introduction animation and the main scene with Pierre. Attractiveness evaluation showed that students were interested about story. However, the ending was too easily predictable: they all imagine the same continuation and ending. We proposed then to complicate the story with sub-stories for maintaining student's interest. Pierre character was not credible enough, so we presented to the public hem many other proposals. One of them got $85 \%$ of acceptation.

Calls window is composed by videos which represent dialogues between Pierre and other characters of the story. Time spent on playing videos was under our expectation, mostly because they were too long and not enough interesting. Basic information was clearly understood, meaning that the mode of transmission was right.

Diary window is composed by images and texts where Pierre explains his quest. Surprisingly for us, time spent by students was over our expectation, mostly because 
Table 4. Zócalo requirements specifications.

\begin{tabular}{|c|c|c|c|}
\hline \multirow{2}{*}{$\begin{array}{c}\text { Communicability attributes } \\
\text { Attractive }\end{array}$} & \multicolumn{2}{|c|}{ Content } & \multirow[t]{2}{*}{ Scene } \\
\hline & Story & Information & \\
\hline \multirow[t]{2}{*}{ Attractiveness } & \multirow{2}{*}{\multicolumn{2}{|c|}{$\begin{array}{l}\text { - Pierre finds Coatlicue commemorative } \\
\text { plate and wonders who she is and where •xplanation of who is Coatlicue } \\
\text { is she }\end{array}$}} & $\begin{array}{l}\text { - Travelling around Zócalo in order to } \\
\text { recognize it, ending at Coatlicue } \\
\text { commemorative plate location }\end{array}$ \\
\hline & & & $\begin{array}{l}\text { - Include typical persons. When user } \\
\text { clicks on them, they talk }\end{array}$ \\
\hline \multirow{5}{*}{ Credibility } & \multirow{5}{*}{$\begin{array}{l}\text { - Information must be true and verified } \\
\text { by experts }\end{array}$} & - Include references and sources & $\begin{array}{l}\text { - Pierre is represented in a realistic style } \\
\text { and students should identify with him }\end{array}$ \\
\hline & & - Include original texts & $\begin{array}{l}\text { - Zócalo is sufficiently realistic for } \\
\text { students being able to recognize it, in the } \\
\text { same style as Pierre }\end{array}$ \\
\hline & & & - Include a moving sky, cars and persons \\
\hline & & & • Zócalo sounds \\
\hline & & & • Buttons should use metaphors \\
\hline
\end{tabular}

Table 5. Main components of prototype to be evaluated.

\begin{tabular}{cclc}
\hline Window & Media & Communicability Metric & Expected Time \\
\hline \multirow{2}{*}{ Zócalo } & Dialogue & Attractiveness questionnaire & -- \\
& Pierre's & Credibility questionnaire & 1 min.30 \\
Animation & Dialogue & Interestingness questionnaire & 30 sec. \\
Diary & Dialogue & Informativeness questionnaire & 1 min. \\
Interactive images & Texts & Interestingness questionnaire & 30 sec. \\
\hline
\end{tabular}

Table 6. Pierre y la coatlicue evaluation.

\begin{tabular}{cccccc}
\hline & $\begin{array}{c}\text { Attractive } \\
(1-5)^{*}\end{array}$ & $\begin{array}{c}\text { Interesting } \\
(1-5)^{*}\end{array}$ & Informative & Credible & Time $^{* *}$ \\
\hline Zócalo & 3.5 & -- & -- & 3 & -- \\
Calls & -- & 3.7 & 5 & -- & 0.5 \\
Diary & -- & 4.2 & 4.1 & -- & 1.5 \\
Photos & -- & 2.5 & -- & -- & 0.7 \\
GPS & -- & 3 & -- & -- & 1.4 \\
\hline
\end{tabular}

"is the best; ${ }^{* *}>1$ is above expectation, $<1$ is under expectation.

texts were very simple and allowed students to understand quickly all information about story.

Photos window did not interest students: they were too small and subjects were not original. We decided to enlarge them and use more original images.

GPS window is composed by the image representing city map. Time spent by students was over our expectation, mostly because they tried to click over all places. However, map was not interactive so it seemed necessary to make it interactive, allowing students to visit virtually the city.

\section{Conclusions}

In this paper, we introduced a methodology for the design of a MIIS, taking into account its communicational aspects. In order to guide the analyst during the specification of the system, we defined a MIIS metamodel that can be used as a domain specific language (DSL). To be able to precisely characterize the MIIS, we complemented the ISO/IEC 25010 model of quality in use with four new quality sub-characteristics of communicability: attractiveness, interestingness, informativeness and credibility. In order to elicit the requirements of a MIIS, without the benefit of the users' and stakeholders' input, we suggested 
the realization of a communicational analysis which derives into the creative concept of MIIS. To deduce the system specification from the creative concept, this has been expressed as a model, instance of the proposed MIIS metamodel and then analyzed it by means of our communication attributes. We have shown with a case study that it is possible to establish the system's specifications quality by analyzing each instance of the class Window of the model through its quality attributes.

Having used it in all along our real case study, we have experienced using the MIIS' metamodel, very useful to describe the system and analyze its quality looking at each of the Windows instances which are all composed of Content and Scene. Communicability attributes enable us to characterize exactly our required quality in use properties and then allow use to test that the system actually meets users' expectations. This work opens new possibilities in taking communication aspects into consideration while designing MIIS. In particular, prototype evaluation used in this work was useful in order to decide if the windows and media defined in the system took actually correctly in charge each communicability attributes and suggesting the way to improve them. This type of evaluations could be performed many times during the iterative development process, assessing how each specific Window was responding to communicability attributes, and this, before the end of the process.

The formalization of a methodology to design a MIIS is becoming more and more indispensable since it offers a multidisciplinary set of communication tools for the use of professionals from very different fields, in one hand, and allows the effective drafting of documents guiding the developers in the other.

\section{REFERENCES}

[1] ISO ISO/IEC 25010:2011, "Systems and Software Engineering-Systems and Software Quality Requirements and Evaluation (SQuaRE)—System and Software Quality Models," 2011.

[2] S. Jones and C. Britton, "Early Elicitation and Definition of Requirements for an Interactive Multimedia Information System,” IEEE Proceedings of ICRE '96, 1996, pp. 12-19.

[3] F. Anthony, "Writing for Visual Media," Focal Press, Waltham, 2006.
[4] B. Davide, G. Franca and P. Paolo, "Value-Driven Design for 'Infosuasive' Web Applications,” World Wide Web Conference, Beijing, 21-25 April 2008, pp. 745-754.

[5] C. Alan, R. Robert and D. Cronin, “About Face 3,” Wiley Publishing, Indianapolis, 2007.

[6] D. Callele, E. Neufeld and K. Schneider, "Emotional Requirements,” IEEE Software, Vol. 25, No. 1, 2008. doi:10.1109/MS.2008.5

[7] D. Zowghi and C. Coulin, "Requirements Elicitation: A Survey of Techniques, Approaches, and Tools," In: A. Aurum and C. Wohlin, Eds., Engineering and Managing Software Requirements, Springer, New York, 2005. doi:10.1007/3-540-28244-0_2

[8] D. Callele, E. Neufeld and K. Schneider, "Requirements Engineering and the Creative Process in the Video Game Industry," Proceedings of the 13th IEEE International Conference on Requirements Engineering (RE'05), 2005.

[9] W. Burleson, A. Gum and I. Harris, "Educational Innovations in Multimedia Systems,” 29th ASEE/IEEE Frontiers in Education Conference, San Juan, 10-13 November 1999.

[10] G. Ruben, “Disciplining Multimedia,” IEEE MultiMedia, July-September 2000, pp. 72-78.

[11] G. Guéneau, "Conduite de Projets en Création Numérique,” Eyrolles, Paris, 2005.

[12] B. León, "Dirección de Documentales Para Televisión. Guión, Producción y Realización,”1a. Edición, Eunesa, 2009.

[13] T. Orehhovacki, "Proposal Set of Quality Attributes for Web 2.0 Application Success," Proceedings of the ITI 32nd Conference on Information Technology Interfaces, Caytat, 21-24 June 2010.

[14] N. Jakob, “Usability Engineering,” Academic Press, Boston, 1994

[15] ISO/IEC TR 9126-4, "Software Engineering-Product Quality_Part 4: Quality in Use Metrics,” 2004.

[16] E. Berki, et al., "Requirements Engineering and Process Modelling in Software Quality Management-Towards a Generic Process Metamodel,” Software Quality Journal, Vol. 12, 2004, pp. 265-283.

[17] S. Nalin, "Authoring Educational Multimedia Content Using Learning Styles and Story Telling Principles," EMME’07, Augsburg, 28 September 2007, pp. 93-102.

[18] R. McKee, "Story: Substance, Structure, Style and the Principles of Screenwriting,” Methuen, London, 1998.

[19] I. Sommerville, “Ingeniería de Software,” 9a. Edición, Pearson Educación, Upper Saddle River, 2011. 